\title{
Extended Classification of the Main Grammatical Frames for Speaking verbs
}

\author{
Nurrahmi ${ }^{*}$ \\ ${ }^{1}$ Department of English Language Studies, Universitas Hasanuddin, Makassar, Indonesia \\ *nurrahmiammy@gmai.com
}

\begin{abstract}
This paper explores further elaboration of grammatical frames of speaking verbs. All data are taken in Corpus of Contemporary American English. This paper aims to 1) To analyse the structure of sentences that refers to the main grammatical frames of Speaking verbs, 2) To extend the classification into the Tense and Aspect category. The research describes the potential category of grammatical frames in Speaking verbs by categorizing and interpreting the structure of the sentence. It is compulsory to use systemic description which adopts deep syntactic analysis: matrix and complement clause, simple and complex sentence, and the use of Tense and Aspect. The extended classification resulted in some classification of grammatical frames; in frame I, 'Describe' is allowed. In frame II, 'Shout' and 'Call' are allowed. In frame III, 'Talk', 'Speak', 'Discuss' and 'Describe' are allowed. In frame IV, 'Talk', 'Discuss', 'Describe' and 'Shout' are included. In frame V, 'Discuss', 'Shout' and 'Call' are included. All classified verbs are also marked in Irrealis, AP (present), PP (present), AP (present) and PP (past). By approaching the data in deep syntactic analysis at the level of clause and sentence, the extended classification of grammatical frames of Speaking verb is also constructed in simple and complex sentence.
\end{abstract}

Key words: Grammatical frame, Speaking verb, tense and aspect.

\section{Introduction}

Grammatical class and Syntactic properties are the principal components of human language that deal with how sentences are constructed, and users employ a striking variety of possible arrangement of the elements in sentence, [1]. Syntactic structure deals with grammar as fundamental lexical properties [2] which aim to categorize words into several dimensions and is formed by grammatical behaviour. In a certain language, verbs are inflected to encode tense, aspect, mood and voice in variable lexemes. Verbs have variable lexemes and some distinct inflectional forms needed in diverse grammatical contexts [3][4], the displaying common properties are the abstraction of verbs displaying some semantic features.

Extended verb classification through semantic approaches have been dominated by scholars in linguistic. Some studies attracted substantial interest of the implementation verb analysis which elaborate the provided data in lexical semantic resources [5][6][7], and combining mix method in classifying grammatical class [8] [9], the studies existed in determining of the progressive verb classification based on lexical semantic aspect and less features of syntactic properties. 
The main implication of syntactic analysis refers to the constituent series of a sentence that is constructed by phrases and word combination by means of grammatical operation [10]. The implementation of syntactic analysis is also applied to different languages; a phrasal category and properties of the noun phrase in Hungarian [11] and verb domain of subject agreement in Turkish [12]. These previous studies have released the advances of syntactic analysis ranging from phrases, clauses, sentences adapted by semantic representation.

The study of the non-action verbs domain particularly a verb that refers to the speaker and addressee needs to be further developed and analysed by taking the data from Corpus Linguistic. In English linguistic, corpus-based study has undergone a notable renaissance in recent years, especially the studies of grammar [13]. The purpose of this present research is analysing the main grammatical frames for Speaking verbs in a sentence, verbal domain has a quite distinct of roles.

The verbal concepts are divided into two categories: primary and secondary, [14] and each type is grouped within a specific subtype that has identical meaning in common. Speaking verb is one of the primary verb types that are divided into some subtypes. Speaking verbs have four semantic roles: The speaker, the addressee, the message, and the medium. The considerable variations will be further discussed considering that there are some limitations of grammatical frames in each subtype at the level of complement clause in the sentences in which the verb exists. Therefore, this study aims: 1) To analyse the structure of sentences referring to main grammatical frames of Speaking verbs, 2) To extend the classification into Tense and Aspect category. This research is expected to the extensive studies in grammatical and lexical semantic in linguistic

\section{Methodology}

The research describes the potential category of grammatical frames in Speaking verb by categorizing and interpreting the structure of the sentence. It is compulsory to use systemic description which adopts deep syntactic analysis: matrix and complement clause, simple and complex sentence, and the use of Tense and Aspect [15] [16] [17], and the feature of grammatical frames in Speaking verb. The data are taken from Corpus of Contemporary American English (COCA).

The data analysis is classified and interpreted to elaborate the research questions to be answered. The steps to be taken as follows: 1) implementation of deep understanding of the text;2) Providing code for each presented data: (1), (2), (3) etc.; 3) The data is classified based on the syntactic properties' construction under Dixon's theory; 4) All classified data is analyzed in deep under syntactic theories to elaborate the structure of sentences in grammatical frames of Speaking verbs at the level of matrix and subordinate clause, simple and complex sentence; 5) All analyzed data is classified based on their Tense and Aspect, those data will be presented in the form of table.

\section{Findings and Discussions}

The data found in COCA for each verb subtype has more than 10,000 text data ranging from $2009-2019$. The data listed consists of spoken, fiction, magazine, newspaper, and academic journals. As formulated in the purpose of this study, it has been addressed that in grammatical frames of Speaking verbs under Dixon's theory have quite distinct rules, some are allowed to fill the object slot and the rest are restricted. There always be questions that assess such a claim and it will be adjusted whether the claim can be expanded or will it remain a perpetual theory.

\section{Main grammatical frames of speaking verbs}

Speaking verbs are divided into some specific subtypes i.e., Talk subtype (talk, speak), Discuss subtype (discuss, refer to, describe), Shout subtype (shout, call), Report subtype (say, state, announce, remark, boast, suggest, undertake, promise), Inform subtype (inform, lecture), Tell subtype, Order subtype (order, forbid), Forgive subtype (forgive, accuse). In this paper, the analysis is limited only to determining Talk, Discuss and Shout subtype possibilities in main grammatical frames for Speaking verbs.

In Speaking verbs, a noun phrase is realised in Speaker, Addressee and Medium; the Message is possible to be put as an NP, complement clause, or direct speech. Speaker role is mapped in subject transitive or intransitive syntactic relation. There is substantial variation ruled and associated with the three components in Speaking verbs.

\section{Message Label}

The message-label is an NP, its head is usually a speech act noun or a noun referring to some language unit and it is linked by a preposition as the second component called the Message-content; an NP, or an Ing, Wh, or That complement clause may are included. This construction is only allowed in Talk subtype, Discuss subtype (except describe), Shout subtype. However, the data shows that describe verb type which is not included as type of verb in this construction, is also existed and the sample are presented below 
Describe.

(1) I cannot describe [the intense feeling] ${ }^{L}$ [you get when you realize this guard has been manned continuously] ${ }^{C}$

(2) I am not going to describe [the scene $]^{L}$ [as I was not there $]^{C}$

As shown in (1) and (2), all construction is formed in complex sentence. Here, describe is followed by the label and message content is added after the label. The components are marked by label (L) and content (C). There are three types of aspects in (1): irrealis, actual perpective and previous perpective while in (2) is marked by irrealis and actual perpective (past)

\section{Content}

A message-content can be a full message, consisting of label, preposition and content or it can be constructed by NP, Ing, Wh-clause function as just a content. In this construction, Talk subtype and Discuss subtype are included while Shout (shout and call) subtype is not included. After some identification regarding this construction, the exception verb type is also set in this construction.

Shout.

(3) We shouted [the whole way] ${ }^{C}$

(4) She shouted [the last, ensuring the boy heard her dire threat] ${ }^{C}$

The component (content) comes after the main verb. (3) and (4) are simple sentences and marked with actual perpective (past). (4) is followed by Ing complement, although this sentence is separated by a comma, it does not mean it is an independent clause because it is connected to the main subject.

Call.

(5) We call [what they want the Dark Reverie]C

(6) Smany people call [what I would call acquaintances friends] ${ }^{C}$

In Call verb type, the construction of the sentence is formed by Wh clause (what). The aspect in (5) is actual perpective (present) and in (6) is marked by actual perpective (present); modal verb appears after the second subject, and it is still marked as actual perpective (present) to express certainty.

\section{That Clause or direct speech}

In this construction shows just Content after a subject, it can be a That clause or a direct speech. This construction is only allowed in Shout subtype. The exception verb type is examined by analysing some possibilities that attest the data provided and the result presented that Talk subtype and Discuss subtype are represented as a series in this construction.

Talk. Most of the sentence structure is formed by much/long/often or directly an NP. However, there are still some sentence constructions which its component indicates that That clause can also be applied to the Talk and Discuss subtype

Call.

(7) [We talk that we are so high and mighty country] ${ }^{M C}$

(8) [We had talked that most likely we will eat dinner somewhere in Restaurant] ${ }^{M C}$

(9) [We often talked that we did not see any more children in our future] ${ }^{M C}$

(10) [[I talked that some people working in the region said [we would be lucky if we're one-third of the way through this right now] $\left.\left.{ }^{S C}\right]\right]^{M C}$

(11) [We talked that we were going to be together ${ }^{M C}$

All That clauses have full structure, subject, object and predicate. (7), (8), (9), (11) are simple sentences sequenced in matrix clause (MC) and (10) is complex sentence with one subordinate clause (SC). The aspect used in (7) is actual perpective while in (8), (9), (10) and (11) are marked by different aspect; in the main verb, (8) is marked by previous perpective (past), (9) - (11) are marked by actual perpective (past).

Speak.

(12) [I do not speak that every evolution is progressive $]^{M C}$

(13) [I speak that I have seen with my father] ${ }^{M C}$

(14) [He warmly spoke that I smiled a bit from my tears] ${ }^{M C}$ 
(15) [He spoke that a preceding call was made before the presidential] ${ }^{M C}$

(16) [[He spoke that I don't really think that [his testimony hurt him or helped him $\left.\left.]^{S C}\right]\right]^{M C}$

(17) [[He spoke that you will overall feel like [you are watching Dr. King] $\left.\left.]^{S C}\right]\right]^{M C}$

The three sentences are marked with one aspect; (12) is actual perpective (present); (14) is actual perpective (past); (15) is actual perpective past (the subordinate verb is in passive form). (13), (16), (17) are marked with two distinct aspects; actual perpective (past) as a head followed by previous perpective (past) in (13); actual perpective (present) in (16); and irrealis status in (17). (12) - (15) are simple sentences that are ordered in matrix clause while (16), (17) are complex sentences.

Discuss. That in this construction is mostly found as a determiner used to indicate which entities are being referred. However, the data below show that That complement is constructed in Discuss subtype

(18) [I will not discuss that I might give away an entry to the event ${ }^{M C}$

(19) [[I will discuss that the vote for statehood happened due to [it is being the choice with the least] $\left.\left.{ }^{S C}\right]\right]^{M C}$

(20) [They discussed that they would celebrate their 2oth wedding anniversary] ${ }^{M C}$

(21) [we have discussed that growing up as a blue eyed Native American leave one in an extremely minority group] ${ }^{M C}$

It is interesting that in (18), (19) are marked as irrealis status as a head followed by actual perpective (past) are being combined in matrix clause; especially in (19) is marked with three aspects that actual imperpective (present) placed as the second subordinate verb. (20) and (21) are simple sentence; (20) is marked by actual perpective (past) with modal auxiliary verb "would"; and (21) is marked by previous perpective (present) with That complement and Ing clause.

Describe.

(22) [It has been recently described that this death can occur in two forms] ${ }^{M C}$

(23) [ [The participants described that they experienced social role changes [when the partner without mental illness began to assume more household responsibilities] $\left.\left.{ }^{S C}\right]\right]^{M C}$

(24) [You described that the president is trying to send the FBI ${ }^{M C}$

(25) [he also described that he had gotten paid \$27 million in doing business with Ukrainians] ${ }^{M C}$

(22) is in passive form marked by previous perpective (present). (22), (24), (25) are simple sentence; (24) is marked by actual perpective (past) and actual imperpective (present) in That complement; (25) is marked by two aspects i.e., actual perpective (past) and previous perpective (past). Complex sentence in (23) is marked by only one aspect in the main and subordinate verbs.

The content in Talk, Discuss and Shout subtype are generally formed with a full structure that modify the main subject and verb. As far as the data is analysed, only That clause can be identified in these verb types, while direct speech remains restricted in certain verbs.

\section{Judgement}

Judgment TO complement has a rather different meaning. The subject of the main clause verb proceeds a judgment or opinion about the subject of the complement clause predicate. State or property which is either transitory or a matter of opinion belong to the Judgment TO and it is used to describe some permanent, objective property. Based on Dixon grammatical frames, the three subtype: Talk, Discover and Shout subtype are not allowed in these frames. However, note that these frames may increase their classification if applied to a wider context or type of text. as in the data showed:

Talk

(26) [you could talk him to read it ${ }^{M C}$

(27) [I might even be able to talk him to take us fishing] ${ }^{M C}$

Speak.

(28) [She tells them that she will speak to him to resolve the matter once] ${ }^{M C}$

(29) [Henry spoke to her to see if she were there] ${ }^{M C}$

In Talk subtype, the judgment TO comes after the object that is linked to the second verb. The component of the sentences is likely to be with modal verb in (26) and (27) in accordance with such an opinion; they are marked in irrealis aspect. (28) is marked by three different aspects and the head is in actual perpective (present) followed by 
irrealis aspect. (29) is marked by actual perpective (past and present) being objective opinion. All the sentences are sequenced in matrix clause

Discuss.

(30) [[We discuss to give her an idea [of how temporary this job might be for me $\left.\left.]^{S C}\right]\right]^{M C}$

(31) [The committee will discuss to determine the future or lack of one, to be more precise] ${ }^{M C}$

(32) [Two hypotheses have been discussed to explain underlying mechanisms] ${ }^{M C}$

Describe.

(33) [You describe to get this working on a 12] ${ }^{M C}$

(34) [[the steps you have describe to achieve the change [make it seem attainable $\left.\left.]^{S C}\right]\right]^{M C}$

The component of Discuss subtype referring to the Judgment TO is mostly constructed with an NP after the main verb. However, the data shows Judgment TO is also acquired in Discuss subtype. (30) and (33) are marked by actual perpective (present) in first and second verb. (31) is marked by irrealis concerning that it is formed by modal verb. (32) and (34) are marked by previous perpective (present); (32) is in passive form which the Speaker is not mentioned and (34) is in active form.

Shout.

(35) [He mentally shouted to cover the previous thought] ${ }^{M C}$

Call.

(36) [We could call to confirm his academic credentials] ${ }^{M C}$

(37) [I called to see what they were worth now ${ }^{M C}$

\section{Modal For To}

Modal (For) TO complement associate to the subject of the complement clause involved in the activity or state pointed to by that clause, or to the potential of such complicity. This construction is not allowed in all these three verb types, Talk, Discuss and Shout subtype. The data found is also still restricted in some subtypes and the extended frames only obtained in Discuss, describe, Shout and call verb type.

Discuss.

(38) [A House Committee will discuss for them to allocate more money] ${ }^{M C}$

(39) [Pipeline analysis and scenarios are discussed for major disease to provide details on the major pipeline developments] ${ }^{M C}$

In Discuss subtype, all sentences are in matrix clause. Describe is still restricted form Modal FOR TO. (38) is marked by irrealis and (39) is marked by actual perpective in passive form.

Shout.

(40) [I will shout for her to join me ${ }^{M C}$

(41) [The kids shout for him to kick the ball back] ${ }^{M C}$

(42) [Danny shouted for him to stop and grabbed at his arm ${ }^{M C}$

Call.

(43) [They never call for you to push start anywhere] ${ }^{M C}$

(44) [The players last month called for the split to change more in favor of the owners] ${ }^{M C}$

(40) is marked by irrealis; (41) and (42) are marked by actual perpective (present and past); (43) is marked by actual perpective (present) and sequenced by adverb of frequency that comes after Subject. (44) is formed by Subject and adverb of time followed by main verb that is marked by actual perpective (past).

The Modal FOR TO is quite similar with TO Infinitive which the second verb must be in the infinitive form. The difference is in the purpose of the second verb and clause; the TO Infinitive is used to express purposes or necessity after a verb followed by NP filling the object slot and Modal FOR TO in main grammatical frames of Speaking verb indicates the addressee is being involved by the Speaker either in accordance with activities or statement. 


\section{Clause and Sentence}

Sentence is built by word that is the smallest syntactically meaningful units. Word, phrase, clause and sentence are accommodated and set up in rank scale.

\section{Matrix clause}

Matrix clause is superordinate clause which does not itself subordinate anything else and it is coextensive with the whole sentence. Matrix clause functions as a label of convenience that refers to the overall clauses in a sentence. In grammatical frames of Speaking verbs, all the sentences are formed with the hierarchical relation between clauses.

\section{Subordinate clause}

Subordinate clause is sometimes placed as a direct or indirect object. The second clause either functions as a content in object slot or the message delivered by the speaker to the addressee. Subordinate clause is always a dependent clause, containing a subject and verb modifying the subject of the main clause. Subordinate clause assists to establish the time sequence, causality and delivering opinion or idea. It is linked by subordinate conjunction to form complex sentence. In grammatical frames of Speaking verb, most of the subordinate clause linked by That complement and Wh complement.

\section{Simple and complex sentence}

If the sentence is only formed by one clause or independent clause, then it categorized as a simple sentence with subject, predicate and object. In Speaking verb, the terms are signed by Speaker as a subject transitive or intransitive, the verbs subtype acted as predicate, and the message and the medium are mapped as object slot. In Speaking grammatical frames, some sentences are formed as simple sentence i.e., (3), (4), (5), (6), (7), (8), (9), (11), (12), (13), (14), (15), (18), (20), (21), (22), (24), (25), (26), (27), (28), (29), (31), (32), (33), (35), (36), (37), (38), (39), (40), (41), (42), (43), and (44).

A complex sentence is formed by one independent clause and dependent clause. It functions to explain or modify the point of the main sentence. As an addition in a dependent clause, it contains an adverbial that specifies time, place, and manner. In the Speaking grammatical frames are found in (1), (2), (10), (16), (17), (19), (23), (30), and (34).

\section{Tense and Aspect}

The location of the state or action in time are marked as a tense. It is divided into past, present or future. Aspect refers to the extension of the state or action in time, which is unitary (perpective), continuous or repeated (imperpective). Mood or modality refers to the reality of the state or location, which is actual (realis), a possibility or a necessity (irrealis). Verbs in Speaking subtypes are acceptable in irrealis, present and past system in its grammatical frames.

The various aspects in Speaking verbs are describe as below: Irrealis status is marked by modals or semi-modals. The main choices include prediction (will and is going to), ability (can and is able to) and necessity (must and has (got) to). Irrealis is found in (1), (2), (18), (19), (26), (27), (31), (36), (38), and (40).

\section{Present}

Present tense is used to describe what is happening, general truth or state, present the opinion. The most commonly grammatical frames of Speaking verbs found in actual perpective:

Actual Perpective. Actual perpective indicates a stative, habitual and true action. It is generally used to indicate an action happens with a time signal or adverb of time. Actual perpective is found in (5), (6), (7), (12), (13), (28), (30), (33), (39), (41), and (43).

Previous perpective. Previous perpective intends to indicate an action that occurred at indefinite time and effects the subject, or an action that occurred many times and has possibility to be repeated in the present, or an action that began in the past and still going on in the present. Previous perpective is found in (21), (22), (32), and (34).

\section{Past}

Past tense refers to states and actions that taking time in the past. It also indicates that the action or state ended in the past

Actual perpective. Actual perpective appoints a finished or completed action/task that take place at a specific point in time in the past. (3), (4), (9), (10), (11), (14), (15), (16), (17), (20), (23), (24), (25), (29), (35), (37), (42), and (44) are marked in actual perpective 
Previous perspective. Previous perpective demonstrates an action that happened before another action in the past. The only construction that is marked by previous perpective is (8).

These findings suggest that main grammatical frames of Speaking verbs can be extended into some updated classification. Considering that there may be changes or renewal of patterns or content over the time and the evolution of the language itself. Certain classifications, particularly in grammatical frames, will change by themselves and deal with semantic features if those features are applied in a wider context. To summarize the occurrence in the various syntactic frames in Speaking verb and the tense and aspect are marked by the verb head in the sentence as shown in the table

Table 1. Extended Classification of Grammatical Frames of Speaking Verbs

\begin{tabular}{|c|c|c|c|c|c|c|c|c|c|c|c|c|}
\hline \multirow[b]{4}{*}{ Verb Subtypes } & \multirow[b]{4}{*}{ Frames } & \multicolumn{11}{|c|}{ Tense and Aspect } \\
\hline & & \multirow[t]{3}{*}{ Irrealis } & \multicolumn{10}{|c|}{ Realis } \\
\hline & & & \multicolumn{2}{|c|}{ Future } & \multicolumn{4}{|c|}{ present } & \multicolumn{4}{|c|}{ Past } \\
\hline & & & Est & Part & AP & Al & PP & $\mathrm{PI}$ & AP & $\mathrm{Al}$ & PP & PI \\
\hline \multicolumn{13}{|l|}{ TALK Subtype } \\
\hline \multirow[t]{5}{*}{ Talk } & 1 & & & & & & & & & & & \\
\hline & II & & & & & & & & & & & \\
\hline & III & & & & $\sqrt{ }$ & & & & $\sqrt{ }$ & & $\sqrt{ }$ & \\
\hline & IV & $\sqrt{ }$ & & & $\sqrt{ }$ & & & & $\sqrt{ }$ & & & \\
\hline & $\mathrm{V}$ & & & & & & & & & & & \\
\hline \multirow[t]{5}{*}{ Speak } & 1 & & & & & & & & & & & \\
\hline & II & & & & & & & & & & & \\
\hline & III & & & & $\sqrt{ }$ & & & & $\sqrt{ }$ & & & \\
\hline & IV & & & & & & & & & & & \\
\hline & $\mathrm{V}$ & & & & & & & & & & & \\
\hline \multicolumn{13}{|c|}{ DISCUSS subtype } \\
\hline \multirow[t]{5}{*}{ Discuss } & 1 & & & & & & & & & & & \\
\hline & II & & & & & & & & & & & \\
\hline & III & $\sqrt{ }$ & & & & & $\checkmark$ & & $\checkmark$ & & & \\
\hline & IV & $\sqrt{ }$ & & & $\sqrt{ }$ & & $\sqrt{ }$ & & & & & \\
\hline & $\mathrm{V}$ & $\sqrt{ }$ & & & $\sqrt{ }$ & & & & & & & \\
\hline \multirow[t]{5}{*}{ Describe } & 1 & $\sqrt{ }$ & & & & & & & & & & \\
\hline & II & & & & & & & & & & & \\
\hline & III & & & & & & $\sqrt{ }$ & & $\sqrt{ }$ & & & \\
\hline & IV & & & & $\sqrt{ }$ & & $\sqrt{ }$ & & & & & \\
\hline & $\mathrm{V}$ & & & & & & & & & & & \\
\hline \multicolumn{13}{|l|}{ SHOUT subtype } \\
\hline \multirow[t]{5}{*}{ Shout } & 1 & & & & & & & & & & & \\
\hline & II & & & & & & & & $\sqrt{ }$ & & & \\
\hline & III & & & & & & & & & & & \\
\hline & IV & $\sqrt{ }$ & & & & & & & $\sqrt{ }$ & & & \\
\hline & V & $\sqrt{ }$ & & & $\sqrt{ }$ & & & & $\sqrt{ }$ & & & \\
\hline \multirow[t]{5}{*}{ Call } & 1 & & & & & & & & & & & \\
\hline & II & & & & $\sqrt{ }$ & & & & & & & \\
\hline & III & & & & & & & & & & & \\
\hline & IV & & & & & & & & & & & \\
\hline & V & & & & $\sqrt{ }$ & & & & $\sqrt{ }$ & & & \\
\hline
\end{tabular}

$\sqrt{ }$ : allowed I) Message-Label, II) Content, III) That clause or direct speech, IV) Judgment TO, V) Modal FOR TO. Est : establish, Part : particular, AP: actual perpective, AI : actual imperpective, PP: previous perpective, PI : previous imperpective.

The table shows some extended classification of grammatical frames; in frame I, Describe verb type is allowed and marked by irrealis. In frame II, Shout is allowed and marked by actual perpective (past); Call is also allowed and marked by AC (present). In frame III, Talk is allowed and marked by AP (present and past) and PP (past); Speak is allowed and marked by AP (past and present); Discuss is marked by irrealis, PP (present) and AP (past); Describe is marked by PP (present) and AP (past). In frame IV, Talk is allowed and marked by irrealis and AP (present and past); Discuss is marked by irrealis, AP and PP (present); Describe is marked by AP and PP (present); Shout is marked by 
irrealis and AP (past). In frame V, Discuss is allowed and marked by irrealis and AP (present); Shout is marked by irrealis and AP (past and present); Call is marked by AP (present and past).

\section{Conclusion}

Verbal system consists of primary and secondary grouped into some very specific subtype that has similar meaning in common. Speaking verb is one of the primary verb types that shared into some subtypes. Speaking verbs have four semantic roles: The speaker, the addressee, the message, and the medium. The considerable variations and limitations have been discussed to extend the grammatical frames classification; I) Message-Label, II) Content, III) That clause or direct speech, IV) Judgment TO, V) Modal FOR TO.

The results show that some extended classification of grammatical frames; in frame I, Describe is allowed. In frame II, Shout and Call are allowed. In frame III, Talk, Speak, Discuss and Describe are allowed. In frame IV, Talk, Discuss, Describe and Shout are included. In frame V, Discuss, Shout and Call are included. All verb types are also marked in Irrealis, AP (present), PP (present), AP (present) and PP (past).

By approaching the data in COCA from a variety of deep syntactic analysis at the level of clause and sentence [15] [16] [17], the extended classification of grammatical frames of speaking verb is also constructed in simple and complex sentences. The constructions are sequenced in matrix clause and complement clause.

\section{References}

[1] R. D and V. V. JR, An Introduction to Syntax. Cambridge University Press, 2004

[2] Andrew Carnie, Syntax: A generative Introduction, Second Edi. Blackwell Publishing Ltd, 2006

[3] Rodney Huddleston and G. K. Pullum, A student's Introduction to English Grammar. Cambridge: Ca, 2005.

[4] B. Aarts and A. McMahon, The Handbook of English Linguistics. Blackwell Publishing Ltd, 2006.

[5] L. Shi and R. Mihalcea, "Putting pieces together: Combining FrameNet, VerbNet and WordNet for robust semantic parsing," Lect. Notes Comput. Sci., vol. 3406, pp. 100-111, 2005, doi: 10.1007/978-3-540-30586-6_9

[6] A. Korhonen and T. Briscoe, "Extended Lexical-Semantic Classification of English Verbs." [Online]. Available: http://www .umiacs.umd.edu/.

[7] S. Leseva and M. Todorova, "Classifying Verbs in WordNet by Harnessing Semantic Resources Bulgarian Senseannotated Corpus View project Dissertation on Lexical Semantics and Argument Realisation of Prefixed Verbs in Bulgarian View project Classifying Verbs in WordNet by Harnessing Semantic Resources." [Online]. Available: https://www.researchgate.net/publication/339618658

[8] D. P. Vinson and G. Vigliocco, "A semantic analysis of grammatical class impairments: semantic representations of object nouns, action nouns and action verbs." [Online]. Available: www . elsevier .com/locate/jneuroling.

[9] L. Fonteyn and S. Hartmann, "Usage-based perspectives on diachronic morphology: A mixed-methods approach towards english ing-nominals," Linguist. Vanguard, vol. 2, no. 1, Dec. 2016, doi: 10.1515/lingvan-20160057.

[10] Abdrew Radford, Analysing English Sentences, A Minimalish Approach. Cambridge: Cambridge University Press, 2009.

[11] M. den Dikken and É. Dékány, "A Restriction on Recursion," Syntax, vol. 21, no. 1, pp. 37-71, Mar. 2018, doi: 10.1111/synt.12149.

[12] G. Güneş, "Morphosyntax and Phonology of Agreement in Turkish," Syntax, vol. 24, no. 2, pp. 143-190, Jun. 2021, doi: 10.1111/synt.12210.

[13] T. McEnery and A. Wilson, Corpus linguistic An Introduction. Edinburgh: Edinburgh University Press., 2001.

[14] R. M. W. Dixon, R. M. W. Dixon, oxford textbooks in linguistics Series editors A Semantic Approach to English Grammar Second Edition. New York: Oxford University Press, 2005. New York: Oxford University Press, 2005.

[15] G. David Morley, Syntax in Functional Grammar: An Introduction to Lexicogrammar in Systemic Linguistics. London: Continium Wellington House, 2000

[16] B. Aarts, English Syntax and Argumentation. New York: PALGRAVE, 2001.

[17] J. Miller, An Introduction to English Syntax. Edinburgh: Edinburgh University Press, 2002. 